\title{
OPIOIDS ARE NOT BETTER THAN NON-OPIOID PAIN MEDICATIONS FOR CHRONIC BACK, HIP, OR KNEE PAIN
}

\author{
STUDY QUALITY: GOOD
}

THE BOTTOM LINE the QR code for details.

Among patients with moderate to severe chronic back pain, or hip or knee osteoarthristis pain, treatment with opioids was not superior to treatment with non-opioid medications for improving pain-related funtion over 12 months.

\section{WHY IS THIS IMPORTANT?}

More than one in ten adults in the USA report chronic pain, ${ }^{1}$ and a high proportion of primary care visits involve the management of chronic pain. ${ }^{2}$ Primary care providers frequently prescribe opioids for treatment of chronic pain despite safety concerns and minimal evidence for efficacy. ${ }^{3}$ Safety concerns surrounding opioid therapy include the development of opioid use disorder and overdose. ${ }^{4}$ While current guidelines discourage opioid prescribing for chronic pain, ${ }^{5}$ randomized trials of opioid therapy reporting long-term pain and function outcomes were lacking. Highquality evidence was needed to prepare providers for discussions with patients regarding chronic pain treatment options.

\section{FACTS}

Structure of the study:

- The Strategies for Prescribing Analgesics Comparative Effectiveness (SPACE) trial ${ }^{6}$ was a randomized, single-blind (to outcome assessors only) trial of 240 patients with moderate to severe chronic back pain or hip or knee osteoarthritis pain who were not already on long-term opioid therapy.
Patients were recruited from Veterans Affairs primary care clinics.

- Of 4485 patients identified with a diagnosis of back or lower extremity pain, 2377 were screened and determined not eligible, and 1843 declined to participate.

- The trial was pragmatic, meaning that patients were enrolled from primary care settings and interventions were delivered with flexibility in medication selection and dosing according to individual patient response. In the opioid group, the first step was immediate-release morphine, oxycodone, or hydrocodone/acetaminophen. In the non-opioid group, the first step was acetaminophen or a non-steroidal, anti-inflammatory drug.

- The outcome measures were pain-related function (the Brief Pain Inventory interference scale over 12 months) and pain intensity (the Brief Pain Inventory severity scale). Both scales average multiple patient-reported items and range from 0 to 10 with higher scores reflecting worse function or increased pain intensity. A 1-point improvement is 
considered clinically meaningful for both scales. Outcomes were assessed every 3 months.

- The adverse outcome was medication-related symptoms measured by a patient-reported checklist (range 0-19).

Findings of the study:

- Improvement in pain-related function did not differ between groups over 12 months (difference 0.1 [ $95 \% \mathrm{Cl},-0.5$ to 0.7$]$ ). Pain intensity was significantly better in the non-opioid group over 12 months, but the difference may not be clinically meaningful (difference $0.5[95 \% \mathrm{Cl}, 0.0-1.0]$ ).

- Adverse medication-related symptoms were significantly more common in the opioid group over 12 months (difference 0.9 [95\% Cl, 0.3-1.5]).

- Secondary outcomes included health-related quality of life, depression symptoms, anxiety symptoms, sleep disturbance, disability from headaches, sexual function, fatigue, and motivation. There were no differences in these outcomes between the two groups except for anxiety, which was improved in the opioid group compared with the non-opioid group. However, it is unclear if this improvement was clinically meaningful.

- Results were similar regardless of the primary pain diagnosis (i.e., back pain or hip or knee osteoarthritis pain).

\section{STUDY QUALITY AND APPLICABILITY}

Of 4485 patients contacted, a considerable number declined to participate (1843, 41\%). Neither patients nor clinicians were blinded; however, outcome assessors were blinded. Study results may not be applicable to non-VA populations (including women). Results do not apply to patients on long-term opioids.

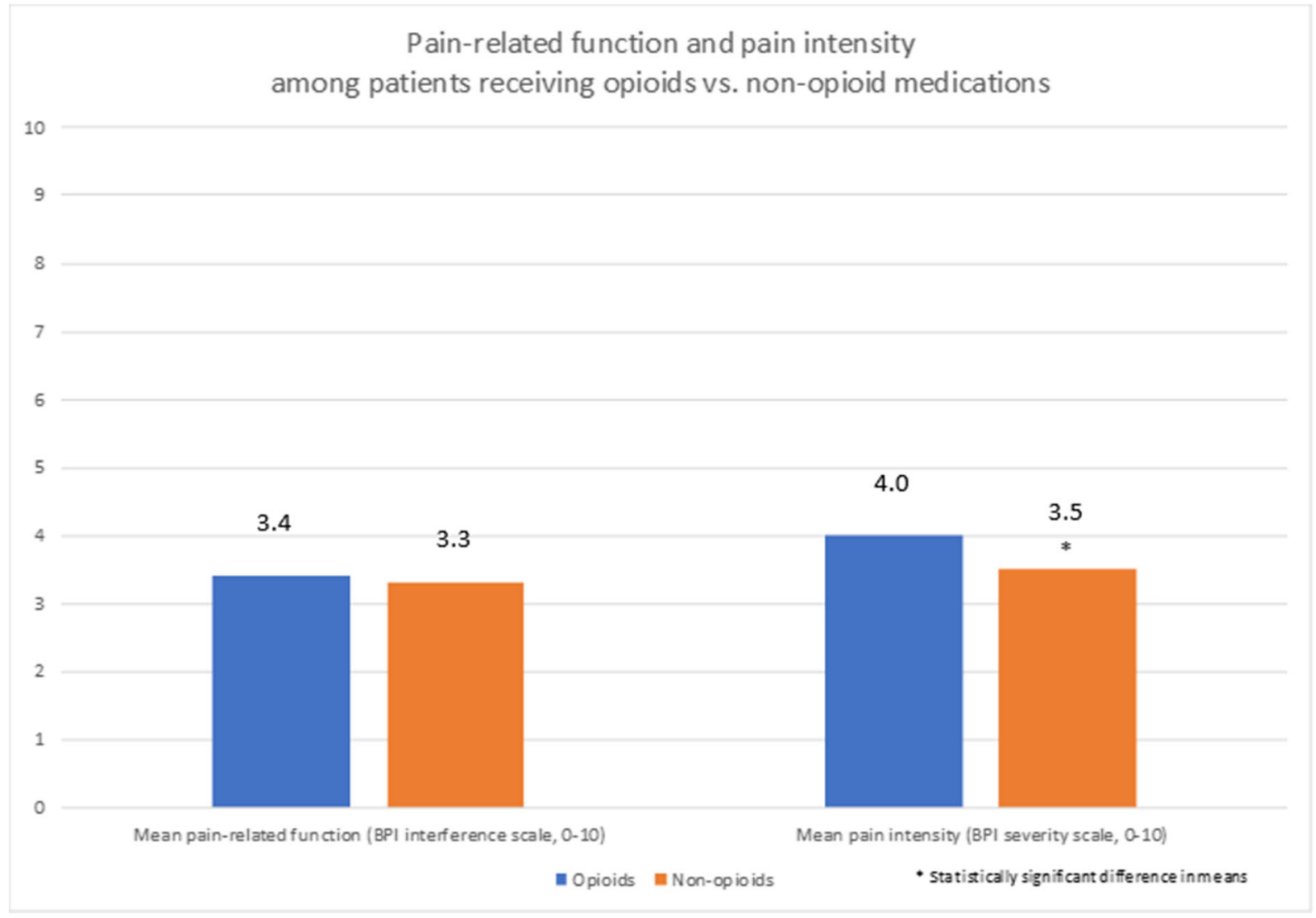




\section{TIPS FOR DISCUSSION OF RESULTS WITH PATIENTS}

- Among patients with chronic back pain, or painful osteoarthritis of the hip or knee, low- or moderate-dose opioids did not lead to better functioning or decreased pain when compared with non-opioid pain medications like acetaminophen or ibuprofen.

- Opioids were associated with more side effects such as memory problems, fatigue, sleep problems, and sexual problems.

- In patients not already on opioids, use of opioid medications for treatment of chronic pain is likely to cause more side effects without noticeable benefits when compared with non-opioid medications.

- Because this trial included mostly men ( $87 \%$ of both groups), it is unclear if the results apply to women.

- This trial did not enroll people already prescribed long-term opioid therapy. The best ways to optimize safety while managing pain in these patients are still being investigated.

\section{AUTHORS}

Corresponding Author: Benjamin J. Oldfield, MD MHS; National Clinician Scholars ProgramYale School of Medicine, New Haven, CT, USA; Department of Medicine, Yale School of Medicine, New Haven, CT, USA (e-mail: benjamin.oldfield@yale.edu)
William S. Fuller, MD; Department of Medicine, Vagelos College of Physicians and Surgeons, Columbia University New York, NY, USA therapy for chronic pain: a systematic review for a National Institutes of Health Pathways to Prevention Workshop. Ann Intern Med. 2015;162(4):276-86. https:// doi.org/10.7326/M14-2559

4. Centers for Disease Control and Prevention. CDC grand rounds: Prescription drug overdoses-a US epidemic. MMWR Morb Mortal Wkly Rep. 2012;61(1):10.
WilliamC. Becker,MD; Department ofMedicine, Yale School of Medicine, New Haven, CT, USA; Pain Research, Informatics, Multimorbidities \& Education (PRIME) Center, VA Connecticut Healthcare System, West Haven, CT, USA

In collaboration with the SGIM Evidence-Based Medicine Subcommittee
5. Dowell D, Haegerich T, Chou R. CDC guideline for prescribing opioids for chronic pain-United States, 2016. MMWR Recomm Rep. 2016;65:1-49.

6. Krebs EE, Gravely A, Nugent S, Jensen AC, DeRonne B, Goldsmith ES, Kroenke K, Bair MJ, Noorbaloochi S. Effect of opioid vs nonopioid medications on painrelated function in patients with chronic back pain or hip or knee osteoarthritis pain. JAMA. 2018;319(9):872.

Publisher's Note: Springer Nature remains neutral with regard to jurisdictional claims in published maps and institutional affiliations. 